\title{
Comparison of Multiscale Method of Cells-Based Models for Predicting Elastic Properties of Filament Wound C/C-SiC
}

\author{
Evan J. Pineda ${ }^{1}$ \\ Marek Fassin ${ }^{2}$ \\ Brett A. Bednarcyk ${ }^{1}$ \\ Stefanie Reese ${ }^{2}$ \\ Jaan-Willem Simon ${ }^{2}$ \\ Paper Number: 175
}

July 2, 2017 


\begin{abstract}
Three different multiscale models, based on the method of cells (generalized and high fidelity) micromechanics models were developed and used to predict the elastic properties of $\mathrm{C} / \mathrm{C}-\mathrm{SiC}$ composites. In particular, the following multiscale modeling strategies were employed: Concurrent multiscale modeling of all phases using the generalized method of cells, synergistic (two-way coupling in space) multiscale modeling with the generalized method of cells, and hierarchical (one-way coupling in space) multiscale modeling with the high fidelity generalized method of cells. The three models are validated against data from a hierarchical multiscale finite element model in the literature for a repeating unit cell of $\mathrm{C} / \mathrm{C}-\mathrm{SiC}$. Furthermore, the multiscale models are used in conjunction with classical lamination theory to predict the stiffness of $\mathrm{C} / \mathrm{C}-\mathrm{SiC}$ plates manufactured via a wet filament winding and liquid silicon infiltration process recently developed by the German Aerospace Institute.
\end{abstract}

\title{
INTRODUCTION
}

Advanced ceramic matrix composites (CMCs) have a wide range of applicability due to the extremely high operating temperatures $\left(>1400^{\circ} \mathrm{C}\right)$ the materials can sustain, as compared to polymer matrix composite (PMC) counterparts, and the improved toughness of CMCs over monolithic ceramics. These attractive features, along with the high specific stiffness and strength, make CMCs ideal for various applications. Moreover, the material behaves more like a structure than a typical material because of the numerous phases, and as such, there is flexibility in designing the material itself. In addition, residual stresses and/or processing induced cracks and voids usually develop during the manufacturing which affect the performance of the CMC.

1. Multiscale and Multiphysics Modeling Branch, NASA Glenn Research Center, 21000 Brookpark Road, MS 49/7, Cleveland, OH 44113

2. Institute of Applied Mechanics, RWTH Aachen University, Mies-van-der-Rohe-Str. 1, 52074, Aachen, Germany 
These manufacturing induced phenomena can be controlled through processing, further expanding the design space for the CMC, and thus, enabling the ability to tailor the material for specific performance criteria, as warranted by a structural design.

A relatively new CMC system of particular interest consists of tows containing carbon $(\mathrm{C})$ fibers embedded in amorphous $\mathrm{C}$ and surrounded by a silicon carbide (SiC) matrix (C/C-SiC) $[1,2]$. C/C-SiC has found utility as an emerging technology for a wide range of applications for various reasons. The ability to tailor and functionally grade the microstructure through processing and manufacturing enables a wider design space of $\mathrm{C} / \mathrm{C}$-SiC structures, and improves the ability to meet structural performance specification [3, 4]. Moreover, C/C-SiC systems exhibit desirable elastic, thermal, fracture, and damage tolerance properties [5, 6, 7]. Finally, the production of $\mathrm{C} / \mathrm{C}-\mathrm{SiC}$ is relatively inexpensive, particularly compared to other CMC systems $[2,8,9]$.

Due to the hierarchical geometric structure and the direct link of the composite performance to the microstructure, micromechanics or multiscale modeling must be employed to accurately capture the response of $\mathrm{C} / \mathrm{C}-\mathrm{SiC}$ structures. Predictive micromechanics and multiscale models of the thermomechanical behavior of $\mathrm{C} / \mathrm{C}-\mathrm{SiC}$ can be effective tools for taking full advantage of the customizable nature of the $\mathrm{C} / \mathrm{C}$ $\mathrm{SiC}$ material system. Blacklock and Hayhurst [10] developed a hierarchical multiscale framework (microscale model is homogenized a priori and output is utilized as input at the meso- or macroscale) for predicting the elastic properties of $\mathrm{C} / \mathrm{C}$-SiC utilizing the finite element method (FEM). Furthermore, several authors have developed analytical micromechanical models to predict the non-linear mechanical response due to damage evolution of $\mathrm{C} / \mathrm{C}-\mathrm{SiC}[11,12,13,14,15,16]$.

It has been demonstrated that filament winding is a promising manufacturing technique, as compared to the use of textile preforms, to attain reinforcement in arbitrary directions within CMC structures [9, 17]. A novel manufacturing method utilizing wet filament wind unidirectional carbon fiber perform and liquid silicon infiltration (LSI) has been develop by the German Aerospace Institute (DLR) to manufacture state-of-the-art $\mathrm{C} / \mathrm{C}$-SiC structures [18]. The filament winding manufacturing process offers increased flexibility in two ways. First, the lay-up of the fiber preform can be tailored to the meet local thermomechanical requirements of the structure. Second, the $\mathrm{C} / \mathrm{C}-\mathrm{SiC}$ microstructure is a function of the filament winding angle [18]. Recently, filament wound $\mathrm{C} / \mathrm{C}-\mathrm{SiC}$ has been used to successfully manufacture a small-scale rocket engine nozzle extension [19].

The analytical micromechanics models lack the ability to directly capture disorder, or non-uniformity, in the arrangement of the constituents of the composites, and often cannot resolve gradients in the local fields (stress and strain), properties, and/or material state. These deficiencies can be overcome with fully-numerical FEM or DEM. Yet, the computational intensity of these models impede their applicability within a synergistic (hierarchical in space, but concurrent in time) multiscale framework. In addition, little (if any) multiscale modeling of filament wound $\mathrm{C} / \mathrm{C}-\mathrm{SiC}$ plates or structures is present in the current literature. Thus, the goal of this current research effort was to develop and evaluate several multiscale models of filament wound $\mathrm{C} / \mathrm{C}-\mathrm{SiC}$ which utilize semi-analytical micromechanics theories that are amenable to a synergistic implementation. 
The method of cells (MoC) family of semi-analytical micromechanics theories, including the generalized method of cells (GMC) and high fidelity generalized method of cells (HFGMC), offer greater fidelity (including local gradients in the fields, properties and material state) than analytical micromechanics models and are more efficient than numerical models [20]. Thus, the MoC theories are suitable for implementation within a synergistic multiscale modeling scheme. Three different multiscale modeling strategies were employed: 1 . Concurrent multiscale modeling of all phases using GMC, herein referred to as "GMC," 2. Synergistic (two-way coupling in space) multiscale modeling with GMC, referred to as "MsGMC" [21], 3. Hierarchical (one-way coupling in space) multiscale modeling with HFGMC, denoted as "MsHFGMC". The three models are validated against results from a hierarchical multiscale FEM model for a repeating unit cell of $\mathrm{C} / \mathrm{C}$-SiC in the available literature [10]. Furthermore, the multiscale models are used in conjunction with classical lamination theory to predict the stiffness of $\mathrm{C} / \mathrm{C}-\mathrm{SiC}$ plates manufactured via a filament winding process recently developed by DLR [22].

The organization of this paper is as follows. The various novel multiscale frameworks developed for $\mathrm{C} / \mathrm{C}$-SiC are presented along with the underlying micromechanics theories. Then, validation results of the multiscale models as compared to data from existing computational results [10] are given. Finally, the multiscale models are used to predict the elastic properties of filament wound plates and compared to experiment.

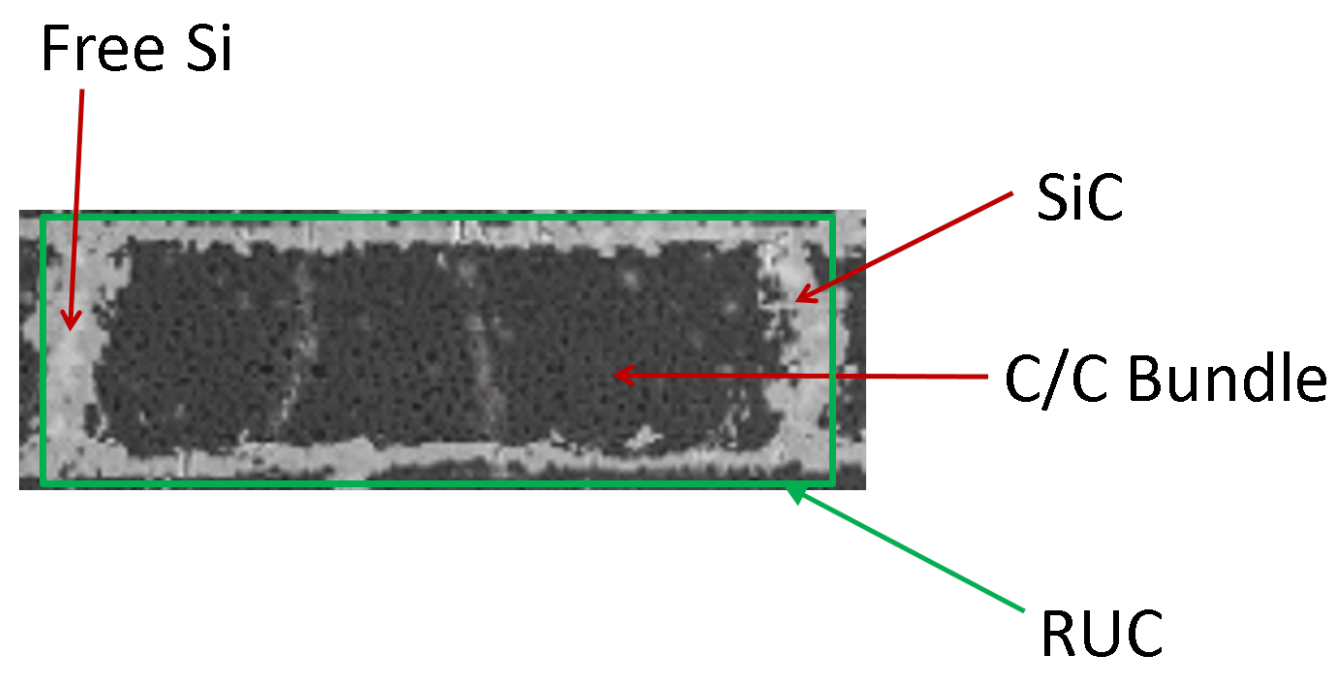

Figure 1. Micrograph of $\mathrm{C} / \mathrm{C}-\mathrm{SiC}$ displaying $\mathrm{RUC}$ with distinct phases: $\mathrm{C}$ fibers within amorphous $\mathrm{C}$ matrix (C/C bundle), $\mathrm{SiC}$ matrix, and free $\mathrm{Si}[22]$. 


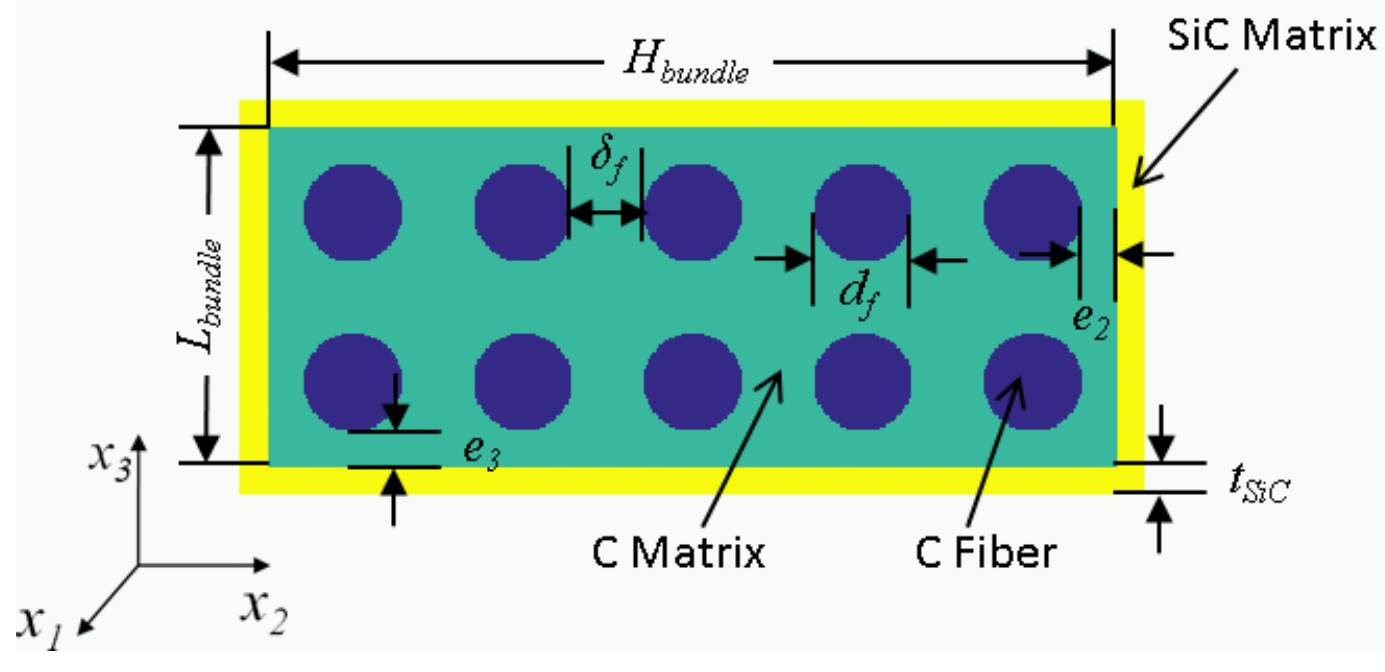

Figure 2. Parameterization of C/C-SiC RUC.

\section{MULTISCALE MODELING OF C/C-SIC RUC}

\section{Parameterization of C/C-SiC RUC}

$\mathrm{C} / \mathrm{C}-\mathrm{SiC}$ composites contain three dominant phases ( $\mathrm{C}$ fiber, amorphous $\mathrm{C}$ matrix, and $\mathrm{SiC}$ matrix), excluding free silicon $(\mathrm{Si})$, existing at various length scales, see Figure 1 [22]. In addition, a repeating unit cell (RUC) within the C/C-SiC microstructure can be readily identified, marked with a green box in Figure 1. These features facilitate the modeling of $\mathrm{C} / \mathrm{C}-\mathrm{SiC}$ via a multiscale strategy. In order to represent the microstructure of the filament wound $\mathrm{C} / \mathrm{C}-\mathrm{SiC}$ composite with an RUC, key features of the microstructure must be identified, idealized, and parameterized. Figure 2 displays the RUC obtained from Figure 1. The $\mathrm{C}$ fibers are shown in dark blue, the amorphous $\mathrm{C}$ matrix is shown in green and the $\mathrm{SiC}$ matrix is shown in yellow. The combination of the $\mathrm{C}$ fibers and amorphous $\mathrm{C}$ matrix is referred to as a " $\mathrm{C} / \mathrm{C}$ bundle." The key parameters within the C/C-SiC RUC are described in Table I. Once these parameters are quantified, along with the volume fraction of each phase, the geometry of the C/C-SiC RUC is completely defined. Following Blacklock and Hayhurst [10], the geometric parameters for the C/C-SiC RUC are given in Table I, and the volume fractions of the constituents are given in Table II. Through homogenization and application of periodic boundary conditions, the response of the RUC represents that of an infinitesimal point in a continuum at a higher scale. Therefore, there is no dependence on length scale, only relative volume fractions of the constituents within the RUC. Hence, the geometric parameters given in Table I are dimensionless.

\section{Concurrent Multiscale Model of C/C-SiC RUC with GMC}

The doubly-periodic formulation of GMC was used to calculate the effective elastic properties of a C/C-SiC RUC, wherein all present phases are modeled concurrently 
TABLE I. LIST AND DESCRIPTION OF KEY MICROSTRUCTURAL PARAMETERS USED TO DEFINE C/C-SIC RUC.

\begin{tabular}{|llc|}
\hline Parameter & Description & Value \\
\hline$H_{\text {bundle }}$ & $x_{2}$ dimension of C/C bundle & 1.0 \\
$L_{\text {bundle }}$ & $x_{3}$ dimension of C/C bundle & 0.15 \\
$d_{f}$ & Diameter of C fiber & 0.029 \\
$\delta_{f}$ & Fiber spacing & 0.0012 \\
$t_{S i C}$ & Thickness of SiC matrix & 0.033 \\
$e_{2}$ & Distance from $x_{2}$ boundary to nearest fiber & 0.0011 \\
$e_{3}$ & Distance from $x_{3}$ boundary to nearest fiber & 0.0007 \\
$n_{2}$ & Number of fibers in $x_{2}$ direction & 27 \\
$n_{3}$ & Number of fibers in $x_{3}$ direction & 4 \\
\hline
\end{tabular}

TABLE II. LIST AND DESCRIPTION OF CONSTITUENT VOLUME FRACTIONS USED TO DEFINE C/C-SIC RUC.

\begin{tabular}{|l|l|c|}
\hline Parameter & Description & Value \\
\hline \hline$v_{C}^{f}$ & C fiber volume fraction & 0.49 \\
\hline$v_{C}^{m}$ & Amorphous C matrix volume fraction & 0.16 \\
\hline$v_{S i C}^{m}$ & SiC matrix volume fraction & 0.35 \\
\hline$v_{C / C}$ & Volume fraction of C/C bundle & 0.65 \\
\hline$v_{C / C}^{f}$ & Volume fraction of C fiber in C/C bundle & 0.76 \\
\hline$v_{C / C}^{m}$ & Volume fraction of amorphous C matrix in C/C bundle & 0.24 \\
\hline
\end{tabular}

at a single scale. GMC, first developed by Paley and Aboudi [23], offers a very computational efficient means of calculating the local fields within and effective properties of a composite RUC. A brief summary of the GMC fundamentals is presented in this section; the reader is referred to Aboudi et al. [20] for complete details on GMC.

With GMC, an RUC must be identified within the composite microstructure, as described in the previous section for the C/C-SiC composites. The RUC is then discretized into a number of rectangular subcells. The $\mathrm{C}$ fiber reinforcement in the $\mathrm{C} / \mathrm{C}$ bundles are continuous in the axial, $x_{1}$-direction. Thus, it is assumed that the RUC extends infinitely in that direction, and the RUC is subjected to a state of generalized plane strain. Therefore, the doubly-periodic formulation of GMC is presented herein. Subcell indices $\beta$ and $\gamma$ are used the location of the subcell within the RUC in the local $x_{2^{-}}$, and $x_{3}$-directions, respectively. Each subcell can be occupied by a single constituent (obeying the constitutive law of that constituent), but there is no limit on the number of different constituents that may be present in the RUC. The displacement fields $\mathbf{u}$ in each subcell $\beta \gamma$ are approximated to be linear.

$$
\mathbf{u}^{(\beta \gamma)}=\mathbf{w}^{(\beta \gamma)}+\bar{x}_{2}^{(\beta)} \boldsymbol{\phi}^{(\beta \gamma)}+\bar{x}_{3}^{(\gamma)} \boldsymbol{\psi}^{(\beta \gamma)}
$$

where $\mathbf{w}^{(\beta \gamma)}$ are the displacement components of the center of the subcells, and $\phi^{(\beta \gamma)}$ and $\psi^{(\beta \gamma)}$ characterize the linear dependence of the displacements on the local subcell coordinates $\bar{x}_{2}$ and $\bar{x}_{3}$. Displacement and traction continuity is enforced in an aver- 
age, or integral sense at each of the subcell interfaces and the periodic boundaries of the RUC. These continuity conditions are used to formulate a strain concentration matrix $\mathbf{A}^{(\beta \gamma)}$, which gives all the local subcell strains $\left(\boldsymbol{\epsilon}^{(\beta \gamma)}\right)$ in terms of the six, global, average, applied strains $\overline{\boldsymbol{\epsilon}}$.

$$
\boldsymbol{\epsilon}^{(\beta \gamma)}=\mathbf{A}^{(\beta \gamma)} \overline{\boldsymbol{\epsilon}}
$$

Noteworthy, even though the formulation is doubly-periodic, the global RUC, and local subcell, stress and strain fields are fully-3D.

Once the strain concentration matrix is formulated and the local subcell strains have been calculated, the local subcell stresses $\left(\boldsymbol{\sigma}^{(\beta \gamma)}\right)$ can be calculated using the local constitutive law and the local subcell strains.

$$
\boldsymbol{\sigma}^{(\beta \gamma)}=\mathbf{C}^{(\beta \gamma)} \boldsymbol{\epsilon}^{(\beta \gamma)}
$$

where $\mathbf{C}^{(\beta \gamma)}$ is the local subcell stiffness, $\boldsymbol{\epsilon}^{(\beta \gamma)}$ contains the six local subcell strains. Eq. (3) can be adjusted to account for both thermal and inelastic strains. However, the analyses considered here are limited to linear elastic materials. Volume weighted averaging can be employed to calculate the average global stresses $\bar{\sigma}$ in terms of the local subcell stresses.

$$
\overline{\boldsymbol{\sigma}}=\frac{1}{H} L \sum_{\beta=1}^{N_{\beta}} \sum_{\gamma=1}^{N_{\gamma}} h_{\beta} l_{\gamma} \boldsymbol{\sigma}^{(\beta \gamma)}
$$

Finally, the global, homogenized RUC stiffness $\mathbf{C}^{*}$ can be calculated.

$$
\mathbf{C}^{*}=\frac{1}{H L} \sum_{\beta=1}^{N_{\beta}} \sum_{\gamma=1}^{N_{\gamma}} h_{\beta} l_{\gamma} \mathbf{C}^{(\beta \gamma)} \mathbf{A}^{(\beta \gamma)}
$$

where $N_{\beta}$, and $N_{\gamma}$ are the total number of subcells in the $x_{2^{-}}$, and $x_{3}$-directions, respectively, $H$, and $L$ are the RUC dimensions in the $x_{2^{-}}$, and $x_{3}$-directions, $h_{\beta}$, and $l_{\gamma}$ are the local subcell dimensions in the $x_{2^{-}}$, and $x_{3}$-directions for subcell $\beta \gamma$.

The RUC used to represent the $\mathrm{C} / \mathrm{C}-\mathrm{SiC}$ microstructure, in a concurrent manner, is shown in Figure 3a, and the associated subcell grid is given in Figure 3b. C fiber subcells are represented in dark blue, amorphous $\mathrm{C}$ matrix in green, and SiC matrix in yellow. This color scheme is used throughout this paper. While the arrangement of fibers in the physical composite is random, a square-packed architecture was chosen as an idealization. Modeling the $\mathrm{C} / \mathrm{C}-\mathrm{SiC}$ with a random microstructure would require statistical considerations when calculating the effective properties. There remained some flexibility in other RUC parameters including number of fibers, fiber diameter, and fiber spacing. A thorough parametric study was conducted to arrive at the RUC displayed in Figure 3. A uniform distribution of fibers is desired because the lack of coupling between normal and shear stresses, inherent in the formulation of GMC, leads to error in the calculation of the local stresses and homogenized properties [24]. Minimization of the difference between $e_{2}$ and $e_{3}$, see Figure 2, represents the most uniform distribution of fibers. It was deemed that the most uniform configuration (smallest difference between $e_{2}$ and $e_{3}$ ) could be achieved with four fibers in the $x_{3}$-direction $\left(n_{3}\right)$ and 27 fibers in the $x_{2}$-direction $\left(n_{2}\right)$, as shown in Figure 3. 


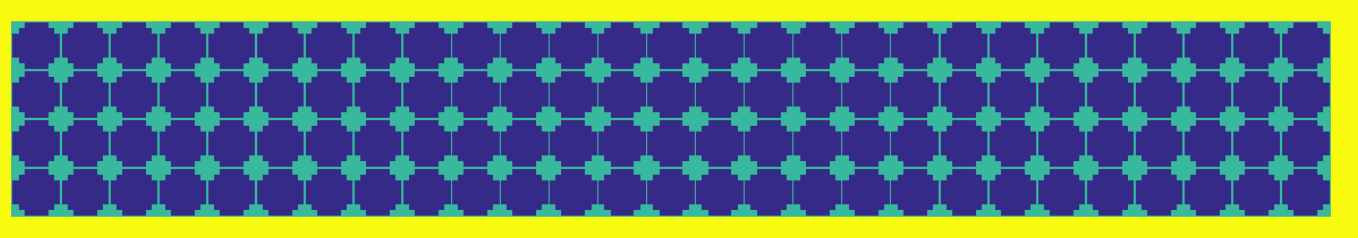

(a) No subcell grid.

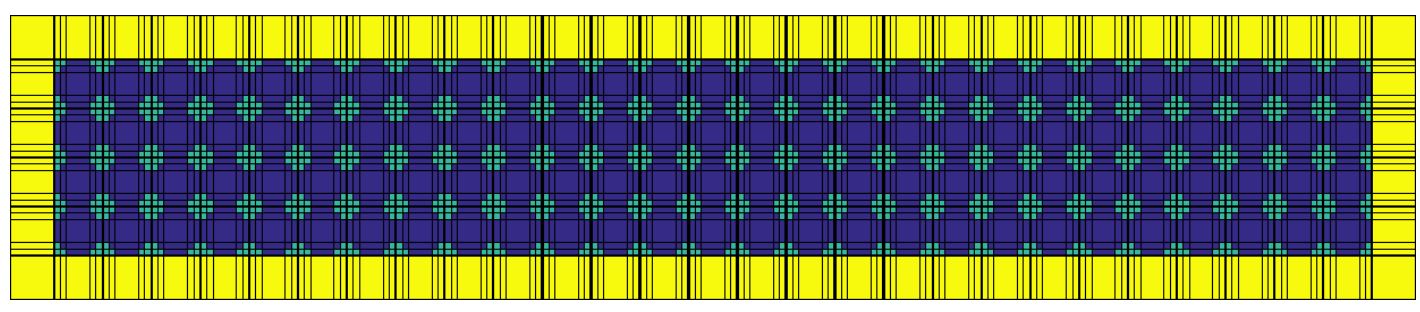

(b) Subcell grid displayed.

Figure 3. Concurrent GMC model of C/C-SiC RUC with C fiber (dark blue), amorphous $\mathrm{C}$ matrix (green), and $\mathrm{SiC}$ matrix (yellow).

\section{Synergistic Multiscale Model of C/C-SiC RUC with MsGMC}

A recursive formulation of GMC, MsGMC, is utilized to model the C/C-SiC RUC across multiple length scales in a synergistic manner (i.e., hierarchical in space but concurrent in time)[21]. MsGMC can admit any general number of scales, or levels, denoted by $i$. Thus, the strain concentration, local stress, and homogenized stiffness relationships given in Eqs. (2)-(5) hold for each level $i$ in the analysis.

$$
\begin{gathered}
\boldsymbol{\epsilon}^{\left(\beta_{i} \gamma_{i}\right)}=\mathbf{A}_{i}^{\left(\beta_{i} \gamma_{i}\right)} \overline{\boldsymbol{\epsilon}}_{i} \\
\boldsymbol{\sigma}_{i}^{\left(\beta_{i} \gamma_{i}\right)}=\mathbf{C}_{i}^{\left(\beta_{i} \gamma_{i}\right)} \boldsymbol{\epsilon}_{i}^{\left(\beta_{i} \gamma_{i}\right)} \\
\overline{\boldsymbol{\sigma}}_{i}=\frac{1}{H} L \sum_{\beta=1}^{N_{\beta}} \sum_{\gamma=1}^{N_{\gamma}} h_{\beta} l_{\gamma} \boldsymbol{\sigma}_{i}^{(\beta \gamma)} \\
\mathbf{C}_{i}^{*}=\frac{1}{H_{i} L_{i}} \sum_{\beta_{i}=1}^{N_{\beta_{i}}} \sum_{\gamma_{i}=1}^{N_{\gamma_{i}}} h_{\beta_{i}} l_{\gamma_{i}} \mathbf{C}_{i}^{\left(\beta_{i} \gamma_{i}\right)} \mathbf{A}_{i}^{\left(\beta_{i} \gamma_{i}\right)}
\end{gathered}
$$

The separate scales are linked by equating the homogenized strains, stresses, and stiffness at Level $i\left(\overline{\boldsymbol{\epsilon}}_{i}, \overline{\boldsymbol{\sigma}}_{i}, \mathbf{C}_{i}^{*}\right.$, respectively) to the local strains, stresses, and stiffness within a given subcell at the next highest scale, Level $i-1\left(\boldsymbol{\epsilon}_{i-1}^{\left(\beta_{i} \gamma_{i}\right)}, \boldsymbol{\sigma}_{i-1}^{\left(\beta_{i} \gamma_{i}\right)}, \mathbf{C}_{i-1}^{\left(\beta_{i} \gamma_{i}\right)}\right.$, respectively).

$$
\overline{\boldsymbol{\epsilon}}_{i}=\boldsymbol{\epsilon}_{i-1}^{\left(\beta_{i-1} \gamma_{i-1}\right)}
$$




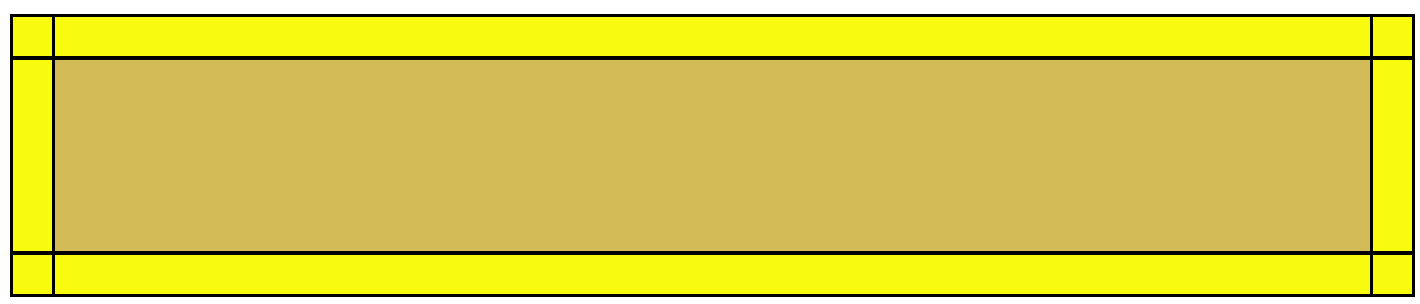

(a) Level 1. Mesoscale: SiC matrix (yellow) and homogenized C/C bundle (gold).

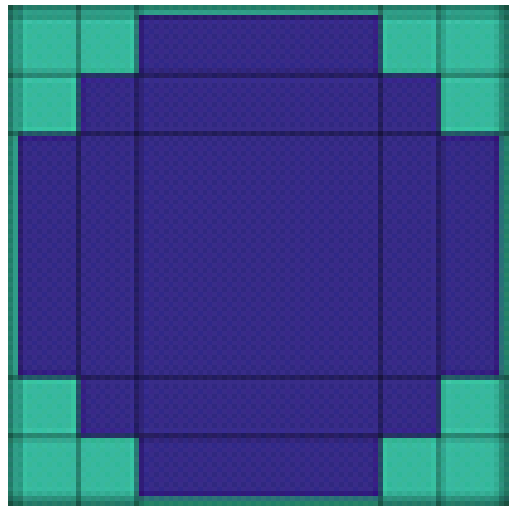

(b) Level 2. Microscale: $\mathrm{C}$ fiber (dark blue) and amorphous $\mathrm{C}$ matrix (green).

Figure 4. MsGMC RUCs and associated subcell grids.

$$
\begin{gathered}
\overline{\boldsymbol{\sigma}}_{i}=\boldsymbol{\sigma}_{i-1}^{\left(\beta_{i-1} \gamma_{i-1}\right)} \\
\mathbf{C}_{i}^{*}=\mathbf{C}_{i-1}^{\left(\beta_{i-1} \gamma_{i-1}\right)}
\end{gathered}
$$

Transformations can be performed such that the lower scale RUC is oriented with respect to a different coordinate system than that of the subcell it is linked to at the higher scale [21]. However, the transformations were omitted from the formulation presented here because all scales utilize the same coordinate-basis vectors. Eqs. (10) - (12) can be used in conjunction with Eqs. (6), (8), and (9) to obtain the homogenized strains, stresses, and stiffness at any scale. Localization and homogenization across any general number of scales is automated.

The C/C-SiC composite is modeled using two levels within MsGMC and the hierarchy shown in Figure 4. At the mesoscale (Level 1) displayed in Figure 4a, the RUC for the C/C-SiC composite is composed of SiC matrix, shown in yellow, and the homogenized $\mathrm{C} / \mathrm{C}$ bundle, shown in gold. Each subcell representing the $\mathrm{C} / \mathrm{C}$ bundle is linked to a microscale (Level 2) RUC, presented in Figure 4b, containing a single $\mathrm{C}$ fiber within amorphous $\mathrm{C}$ matrix depicting a square packed architecture.

The geometry used to represent the fiber, shown in Figure $4 b$, was chosen after a thorough convergence study. For a fixed geometry, the local and global results are completely insensitive to refinements in the subcell grid used to represent the geometry because of the lack of normal-shear coupling in GMC. Thus, the grids 
shown in Figure 4 represent the minimum level of discretization needed to represent the chosen geometry.

\section{Hierarchical Multiscale Model of C/C-SiC RUC with MsHFGMC}

GMC offers a computationally efficient platform for homogenization of composite microstructures, and provides reasonable accurate results for effective properties and other averaged quantities. Conversely, HFGMC offers more accurate local fields at a computational expense, but attains normal-shear coupling. A third model was devised, MsHFGMC, which utilizes the HFGMC micromechanics theory across multiple scales to model the $\mathrm{C} / \mathrm{C}-\mathrm{SiC}$ composite . However, unlike MsGMC, the scale bridging has not yet been automated (synergistic). Instead, homogenization is performed in a "bottom-up," one-way coupling hierarchical scheme. It should be noted that, there exists no difference between the homogenized composite stiffness results obtained from hierarchical and synergistic multiscale models for a linear analysis, like that performed herein. In this section, a brief overview of the HFGMC micromechanics theory is given, and the reader is referred to Aboudi, et al. for more details [20].

HFGMC utilizes quadratic approximations of the displacement fields in each subcells at Level $i$.

$$
\begin{aligned}
\mathbf{u}_{i}^{\left(\beta_{i} \gamma_{i}\right)}= & \overline{\boldsymbol{\epsilon}}_{i} \mathbf{y}_{i}+\mathbf{W}_{i(00)}^{\left(\beta_{i} \gamma_{i}\right)}+\bar{x}_{2_{i}}^{\left(\beta_{i}\right)} \mathbf{W}_{i(01)}^{\left(\beta_{i} \gamma_{i}\right)}+\bar{x}_{3_{i}}^{\left(\gamma_{i}\right)} \mathbf{W}_{i(01)}^{\left(\beta_{i} \gamma_{i}\right)}+ \\
& \frac{1}{2}\left(3 \bar{x}_{2_{i}}^{\left(\beta_{i}\right)^{2}}-\frac{h_{\beta_{i}}^{2}}{4}\right) \mathbf{W}_{i(20)}^{\left(\beta_{i} \gamma_{i}\right)}+\frac{1}{2}\left(3 \bar{x}_{3_{i}}^{\left(\gamma_{i}\right)^{2}}-\frac{l_{\gamma_{i}}^{2}}{4}\right) \mathbf{W}_{i(02)}^{\left(\beta_{i} \gamma_{i}\right)}
\end{aligned}
$$

where $\overline{\boldsymbol{\epsilon}}_{i}$ are the externally applied average strain components, $\mathbf{W}_{i(00)}^{\left(\beta_{i} \gamma_{i}\right)}$ are the volumeaveraged displacements, and $\mathbf{W}_{i(m n)}^{\left(\beta_{i} \gamma_{i}\right)}$ (when both $m \neq 0$ and $n \neq 0$ ) characterize the quadratic dependence of the displacements on the local subcell coordinates. The unknown terms $\mathbf{W}_{i(m n)}^{\left(\beta_{i} \gamma_{i}\right)}$ are determined through satisfaction of equilibrium, enforcement of periodic boundary conditions and continuity of displacements and tractions at the subcell interfaces. As with GMC, continuity conditions are satisfied in an average (integral) sense.

Imposing these conditions at each level (scale) yields a linear set of algebraic equations.

$$
\mathbf{K}_{i} \mathbf{U}_{i}=\mathbf{f}_{i}
$$

The $\mathbf{K}_{i}$ matrix contains geometric and stiffness terms for the individual subcells at Level $i$, the displacement vector $\mathbf{U}_{i}$ is composed of the unknown displacement coefficients (i.e., components of $\mathbf{W}_{i(m n)}^{\left(\beta_{i} \gamma_{i}\right)}$ ) at Level $i$, and the force vector $\mathbf{f}_{i}$ consists of the applied average strains $\overline{\boldsymbol{\epsilon}}_{i}$ ay Level $i$. A localization relationship, similar to Eq. (6) for GMC, between the applied average strains and the local subcell strains at Level $i$ can be obtained by solving Eq. (14).

$$
\boldsymbol{\epsilon}_{i}^{\left(\beta_{i} \gamma_{i}\right)}=\mathbf{A}_{H F_{i}}^{\left(\beta_{i} \gamma_{i}\right)} \overline{\boldsymbol{\epsilon}}_{i}
$$

where $\mathbf{A}_{H F_{i}}^{\left(\beta_{i} \gamma_{i}\right)}$ is the HFGMC strain concentration matrix at Level $i$. Once the strain concentration matrix has been calculated, the homogenized stiffness at Level $i$ can be 
readily determined.

$$
\mathbf{C}_{i}^{*}=\frac{1}{H_{i} L_{i}} \sum_{\beta_{i}=1}^{N_{\beta_{i}}} \sum_{\gamma_{i}=1}^{N_{\gamma_{i}}} h_{\beta_{i}} l_{\gamma_{i}} \mathbf{C}_{i}^{\left(\beta_{i} \gamma_{i}\right)} \mathbf{A}_{H F_{i}}^{\left(\beta_{i} \gamma_{i}\right)}
$$

Since the scale bridging is not automated, the local fields in MsHFGMC are not calculated, and handshaking across the scales is achieved in a bottom-up hierarchical manner with Eq. (12).

The MsHFGMC model operates at the same two length scales, defined in Figure 5, as the MsGMC model described previously. The same geometry at Level 1 was used to represent the $\mathrm{SiC}$ matrix and homogenized $\mathrm{C} / \mathrm{C}$ bundle in both MsGMC and MsHFGMC models. Unlike GMC, HFGMC is sensitive to refinements in the subcell grid. A convergence study was performed to determine the appropriate subcell grid density for Level 1 shown in Figure 5a. Finally, a study on both refinement in the fiber geometry and subcell gird were performed to arrive at the RUC for the $\mathrm{C} / \mathrm{C}$ bundle displayed in Figure 5b.

\section{NUMERICAL VALIDATION OF MULTISCALE MODELS OF C/C-SIC RUC}

In this section predictions for the elastic properties of $\mathrm{C} / \mathrm{C}-\mathrm{SiC}$ obtained using the three models presented in the preceding section are validated against results from

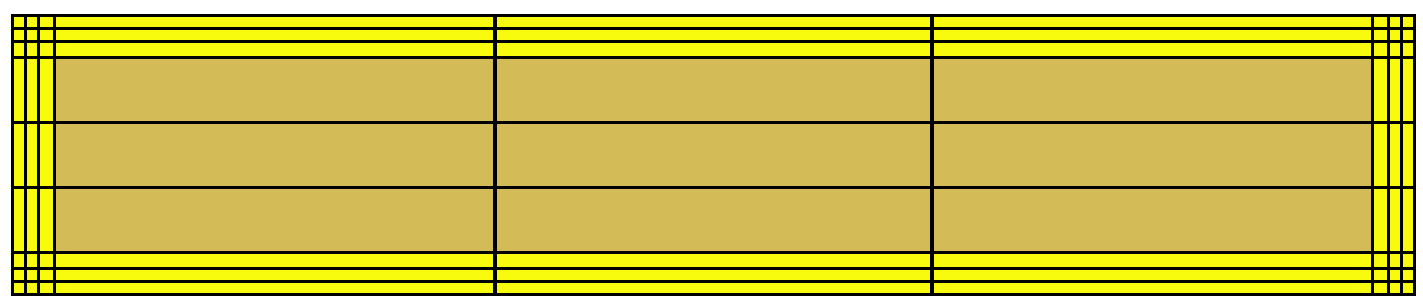

(a) Level 1. Mesoscale: SiC matrix (yellow) and homogenized C/C bundle (gold).

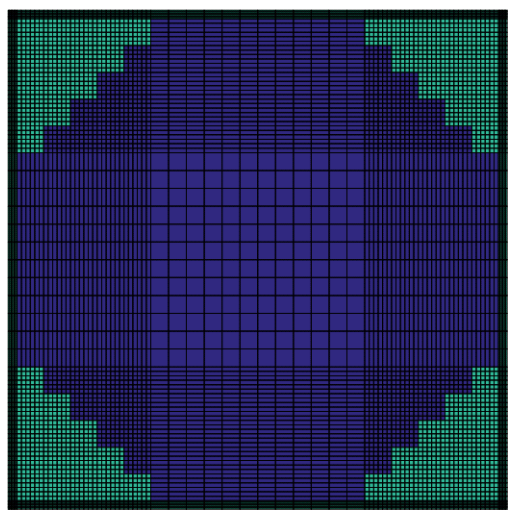

(b) Level 2. Microscale: $\mathrm{C}$ fiber (dark blue) and amorphous C matrix (green).

Figure 5. MsHFGMC RUCs and associated subcell grids. 
TABLE III. ELASTIC PROPERTIES FOR C FIBER AND AMORPHOUS C MATRIX USED IN MULTISCALE MODELS.

\begin{tabular}{|l|c|l|c|}
\hline C Fiber & Value & Amorphous C Matrix & Value \\
\hline \hline$E_{11}^{C_{f}}(\mathrm{GPa})$ & 294.0 & $E^{C_{m}}(\mathrm{GPa})$ & 62.6 \\
\hline$E_{22}^{C_{f}}(\mathrm{GPa})$ & 18.4 & $\nu^{C_{m}}$ & 0.3 \\
\hline$\nu_{12}^{C_{f}}$ & 0.2 & $G^{C_{m}}(\mathrm{GPa})$ & 24.0 \\
\hline$\nu_{23}^{C_{f}}$ & 0.25 & & \\
\hline$G_{12}^{C_{f}}(\mathrm{GPa})$ & 8.96 & & \\
\hline
\end{tabular}

TABLE IV. ELASTIC PROPERTIES FOR SIC MATRIX USED IN MULTISCALE MODELS.

\begin{tabular}{|l|c|l|c|}
\hline Damaged SiC Matrix & Value & Undamaged SiC Matrix & Value \\
\hline \hline$E_{11}^{S i C_{m}}(\mathrm{MPa})$ & 0.001 & $E^{S i C_{m}}(\mathrm{GPa})$ & 15.0 \\
\hline$E_{22}^{S i C_{m}}(\mathrm{GPa})$ & 15.0 & $\nu^{S i C_{m}}$ & 0.3 \\
\hline$\nu_{12}^{S i C_{m}}$ & $2.0 \mathrm{E}-8$ & $G^{S i C_{m}}(\mathrm{GPa})$ & 5.77 \\
\hline$\nu_{23}^{S i C_{m}}$ & 0.3 & & \\
\hline$G_{12}^{S i C_{m}}(\mathrm{GPa})$ & 5.77 & & \\
\hline
\end{tabular}

a bottom-up, hierarchical multiscale FEM model of C/C-SiC tows within a woven composite [10]. The RUCs modeled in this work utilized the same geometrical and volume fraction data (given in Tables I and II), although the intent of the current work is to predict the elastic properties of $\mathrm{C} / \mathrm{C}-\mathrm{SiC}$ plates manufactured using a wet filament winding technique. In addition, the elastic properties for the $\mathrm{C}$ fiber, amorphous $\mathrm{C}$ matrix, and $\mathrm{SiC}$ matrix constituents were also taken from Blacklock and Hayhurst [10] and given in Tables III and IV.

In Blacklock and Hayhurst [10] it was stated that cracks developed in the $\mathrm{SiC}$ matrix during processing. As such, the value for the SiC stiffness in the direction aligned with the fiber axis $E_{11}^{S i C_{m}}$, used in the FEM model was chosen to be zero . There is no mention of how the other properties were treated; so, it is assumed that these other properties did not change from the undamaged isotropic values, yielding an anisotropic stiffness matrix for the cracked SiC. However, in the doubly-periodic formulation of GMC and HFGMC, used for the analyses presented here, it is assumed that the components of the stiffness matrix are transversely isotropic. Thus, if the value for the axial Poisson's ratio $\nu_{12}^{S i C_{m}}$ was not set to a very low value, as listed in Table IV, some of the resulting Young's moduli for the homogenized C/C-SiC RUC would be non-physical (i.e., negative).

The results for all six engineering constants for the C/C-SiC RUC predicted using GMC, MsGMC, and MsHFGMC are presented in Table $\mathrm{V}$ along with the percentage difference from the FEM predictions of Blacklock and Hayhurst [10]. MsHFGMC predicted elastic properties closest to the FEM analysis for nearly all properties, followed by MsGMC and the GMC. The predictions from the models matched most closely to the FEM for the Young's moduli, exhibiting a $\sim 0-10 \%$ difference, and the predictions for the shear moduli were within $\sim 3-18 \%$ of the FEM. A significant dis- 
TABLE V. ELASTIC PROPERTIES OF C/C-SIC RUC PREDICTED WITH MULTISCALE MOC MODELS, INCORPORATING DAMAGED SIC, COMPARED TO MULTISCALE FEM CALCULATIONS FROM BLACKLOCK AND HAYURST, 2012.

\begin{tabular}{|l|r|r|r|r|r|r|}
\hline Property & GMC & $\begin{array}{r}\text { \% Difference } \\
\text { from FEM }\end{array}$ & MsGMC & $\begin{array}{r}\text { \% Difference } \\
\text { from FEM }\end{array}$ & MsHFGMC & $\begin{array}{r}\text { \% Difference } \\
\text { from FEM }\end{array}$ \\
\hline \hline$E_{11}(\mathrm{GPa})$ & 155.2 & 0.6 & 155.2 & 0.6 & 155.2 & 0.6 \\
\hline$E_{22}(\mathrm{GPa})$ & 20.5 & 6.4 & 20.7 & 5.5 & 21.3 & 2.7 \\
\hline$E_{33}(\mathrm{GPa})$ & 19.6 & 9.3 & 20.1 & 7.0 & 21.6 & 4.9 \\
\hline$\nu_{12}$ & 0.167 & 34.4 & 0.168 & 34.0 & 0.173 & 32.0 \\
\hline$\nu_{13}$ & 0.143 & 45.2 & 0.146 & 44.2 & 0.149 & 43.1 \\
\hline$\nu_{23}$ & 0.272 & 12.4 & 0.268 & 13.8 & 0.272 & 12.5 \\
\hline$G_{23}(\mathrm{GPa})$ & 7.45 & 8.9 & 7.45 & 8.9 & 7.94 & 2.8 \\
\hline$G_{13}(\mathrm{GPa})$ & 8.34 & 18.3 & 8.44 & 17.2 & 8.60 & 15.7 \\
\hline$G_{12}(\mathrm{GPa})$ & 8.96 & 12.1 & 9.01 & 11.6 & 9.25 & 9.3 \\
\hline
\end{tabular}

TABLE VI. ELASTIC PROPERTIES OF C/C-SIC RUC PREDICTED WITH MULTISCALE MOC MODELS, INCORPORATING UNDAMAGED SIC, COMPARED TO MULTISCALE FEM CALCULATIONS FROM BLACKLOCK AND HAYURST, 2012.

\begin{tabular}{|l|r|r|r|r|r|r|}
\hline Property & GMC & $\begin{array}{r}\text { \% Difference } \\
\text { from FEM }\end{array}$ & MsGMC & $\begin{array}{r}\text { \% Difference } \\
\text { from FEM }\end{array}$ & MsHFGMC & $\begin{array}{r}\text { \% Difference } \\
\text { from FEM }\end{array}$ \\
\hline \hline$E_{11}(\mathrm{GPa})$ & 160.0 & 3.9 & 160.0 & 3.9 & 160.0 & 3.8 \\
\hline$E_{22}(\mathrm{GPa})$ & 21.0 & 4.0 & 21.3 & 3.0 & 21.9 & 0.3 \\
\hline$E_{33}(\mathrm{GPa})$ & 20.5 & 5.0 & 21.1 & 2.6 & 21.6 & 0.2 \\
\hline$\nu_{12}$ & 0.251 & 1.3 & 0.251 & 1.2 & 0.254 & 0.0 \\
\hline$\nu_{13}$ & 0.255 & 2.2 & 0.258 & 1.2 & 0.261 & 0.0 \\
\hline$\nu_{23}$ & 0.312 & 0.3 & 0.308 & 1.1 & 0.312 & 0.2 \\
\hline$G_{23}(\mathrm{GPa})$ & 7.45 & 8.87 & 7.45 & 8.9 & 7.94 & 2.8 \\
\hline$G_{13}(\mathrm{GPa})$ & 8.34 & 18.3 & 8.44 & 17.2 & 8.60 & 15.7 \\
\hline$G_{12}(\mathrm{GPa})$ & 8.96 & 12.1 & 9.01 & 11.6 & 9.25 & 9.3 \\
\hline
\end{tabular}

crepancy $(\sim 12-45 \%)$ between the Poisson's ratios predicted here and that from the FEM model was observed. This can be attributed to the value for axial Poisson's ratio for the damaged $\mathrm{SiC}$ matrix $\nu_{12}^{S i C_{m}}$ that was needed, see Table IV, because of the assumption of transverse isotropy in the double-periodic MoC-based formulations. If it is assumed the $\mathrm{SiC}$ matrix in the MoC-based models is undamaged and isotropic properties are used, a drastic reduction to $0-2 \%$ in difference between the Poisson's ratios of the $\mathrm{C} / \mathrm{C}-\mathrm{SiC}$ RUC predicted using the current models and the FEM model in the literature is observed, as shown in Table VI.

All multiscale simulations were executed on a single $2.40 \mathrm{GHz}$ Intel Xeon E5$2630 \mathrm{v} 3$ processor (cpu). Figure 6 shows the analysis time versus total number of subcells for each model. The run time increases with the total number of subcells in the model with MsGMC being the fastest. However, all analyses completed in less than 10 seconds. 


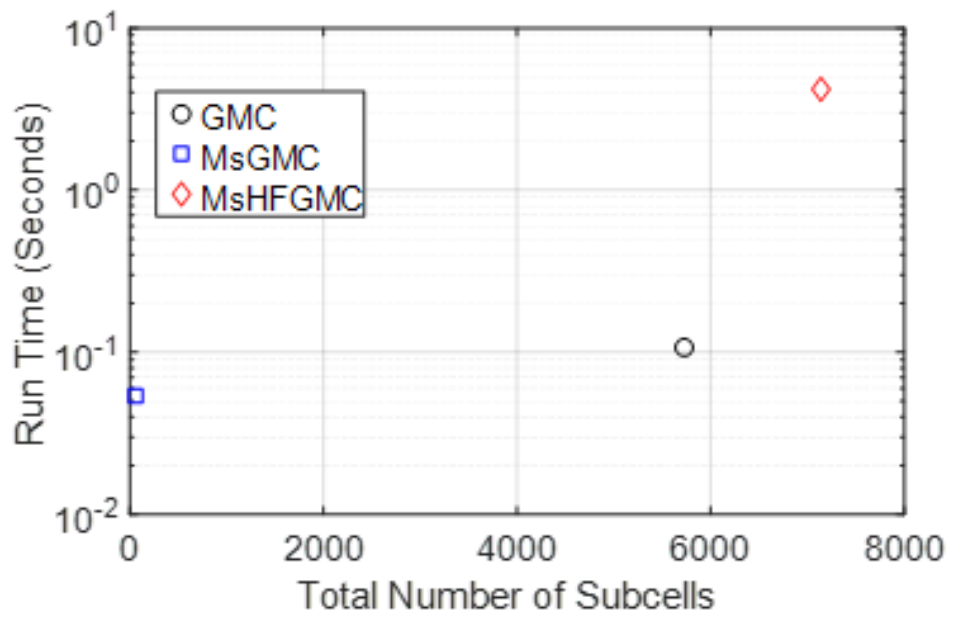

Figure 6. Analysis time versus total number of subcells for three multiscale models of C/C$\mathrm{SiC}$.

The results given in Tables V and VI show that GMC, MsGMC, and MsHFGMC, can predict elastic properties for a $\mathrm{C} / \mathrm{C}-\mathrm{SiC}$ that are comparable to previously published FEM results and serve as a numerical validation of these methods. Herein, subsequent results will be normalized to experimental data or baseline results from the MoC-based models.

\section{RESULTS AND DISCUSSION}

A focus of this paper is to utilize the MoC-based models (GMC, MsGMC, and MsHFGMC) to predict the elastic properties of C/C-SiC plates, manufactured by Breede and colleagues using a wet filament winding and LSI technique, where it has been shown that the $\mathrm{C} / \mathrm{C}-\mathrm{SiC}$ microstructure is dependent on the filament winding angle [18]. Unfortunately, significant data on the geometry and volume fraction of the constituents within the filament wound $\mathrm{C} / \mathrm{C}-\mathrm{SiC}$, needed to create a representative RUC, was not available. Therefore, the same RUC used for the predictions in the previous section with the geometry and constituent volume fractions given in Tables I and II for a tow within a woven $\mathrm{C} / \mathrm{C}$-SiC material are used to predict the elastic properties of filament wound $\mathrm{C} / \mathrm{C}-\mathrm{SiC}$. The elastic properties for the constituent materials presented in Tables III and IV are also used. To model the filament wound $\mathrm{C} / \mathrm{C}-\mathrm{SiC}$ plates, the homogenized response the $\mathrm{C} / \mathrm{C}-\mathrm{SiC} \mathrm{RUC}$ is used to represent the behavior of the layers within a $[\theta /-\theta]$ laminate using classical lamination theory (CLT), where $\theta$ is the filament winding angle [25]. The available reports did not comment if the filament wound plates were manufactured to have a symmetric configuration. Yet, it is assumed here that the plates are symmetric to avoid coupling between axial normal loads and shear loads or moments during coupon testing.

Three different filament winding configurations $\left(\left[0^{\circ} / 90^{\circ}\right],\left[15^{\circ} /-15^{\circ}\right]\right.$, and $\left[45^{\circ} /\right.$ $45^{\circ}$ ]) were manufactured and tested by Breede et al. [22]. Figure 7 displays the 


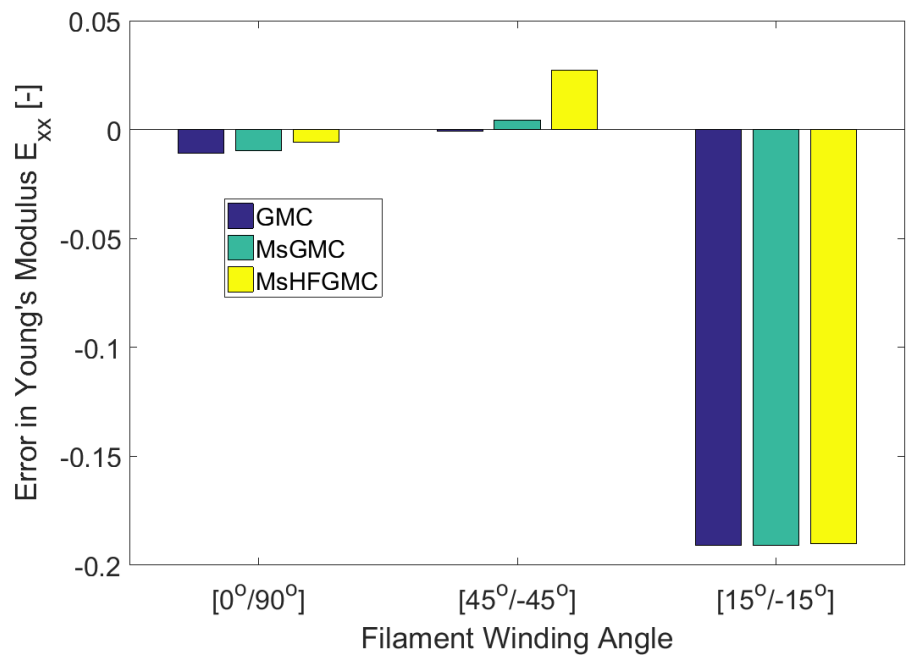

Figure 7. Prediction of Young's modulus $E_{x x}$ of filament wound $\mathrm{C} / \mathrm{C}-\mathrm{SiC}$ at various winding angles compared to experiment from Breede et al. [22].

predicted values for the Young's moduli in the loading direction $E_{x x}$ of the coupons, normalized to the experimental mean, for three different filament winding angles $(\theta)$. The stiffness predictions for the $\left[0^{\circ} / 90^{\circ}\right]$ and $\left[45^{\circ} / 45^{\circ}\right]$ configurations showed excellent agreement ( $<3 \%$ error) with the experimental data, and are fairly consistent across all three multiscale models. Conversely, all the models exhibit $\sim 20 \%$ error when predicting the stiffness of the $\left[15^{\circ} /-15^{\circ}\right]$ plate. Substantial differences between the microstructures of the $\left[45^{\circ} /-45^{\circ}\right]$ and $\left[15^{\circ} /-15^{\circ}\right]$ plates were reported by Breede et al. [22]. Thus, it is inferred that the idealization of the microstructure of the tows within the woven $\mathrm{C} / \mathrm{C}-\mathrm{SiC}$ more closely represents that of the $\left[0^{\circ} / 90^{\circ}\right]$ and $\left[45^{\circ} /-45^{\circ}\right]$ filament wound $\mathrm{C} / \mathrm{C}-\mathrm{SiC}$, whereas new geometric information would be required to accurately predict the properties of the $\left[15^{\circ} / 15^{\circ}\right]$ filament wound $\mathrm{C} / \mathrm{C}-\mathrm{SiC}$ using the MoC-based multiscale models.

It is expected that multi-axial stress states will be present throughout the filament wound structure during service. Thus, the transverse stiffness $E_{y y}$ and shear stiffness $G_{x y}$ of the filament wound plates are also important. Figure 8 shows predictions of $E_{x x}, E_{y y}$, and $G_{x y}$ for all three filament winding angles obtained with all three multiscale models. From these predictions, it can be deduced that the $\left[0^{\circ} / 90^{\circ}\right]$ has an intermediate axial and transverse stiffness but low shear stiffness, as compared to the other lay-ups. The $\left[15^{\circ} / 15^{\circ}\right]$ exhibits the highest axial stiffness, but low transverse and shear stiffness. Finally, the $\left[45^{\circ} / 45^{\circ}\right]$ offers the highest shear stiffness. These stiffness predictions can be utilized in the preliminary design phase of a $\mathrm{C} / \mathrm{C}$ $\mathrm{SiC}$ structure to meet the stiffness requirements throughout the structure under local, multi-axial stress states. In addition, with the appropriate data, the microstructure in the different lay-ups can also be modeled as a function of the filament winding angle. 


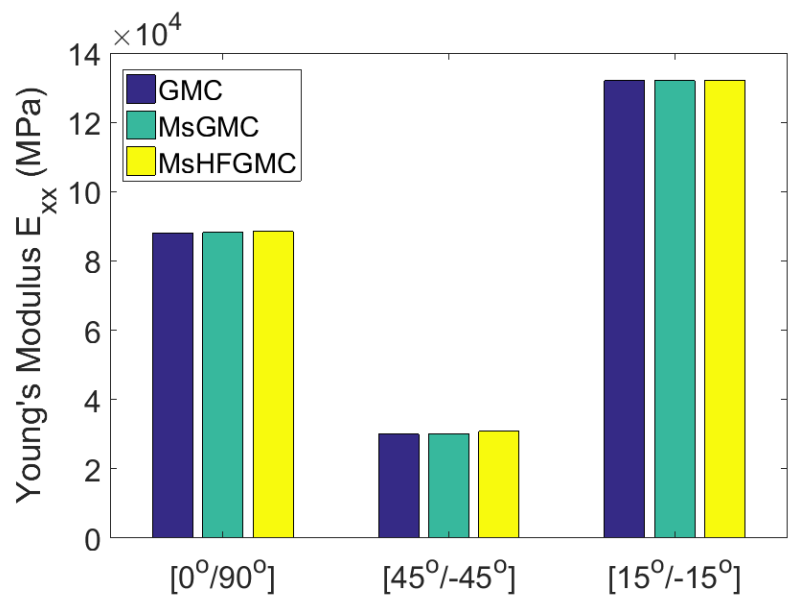

Filament Winding Angle

(a) $E_{x x}$.

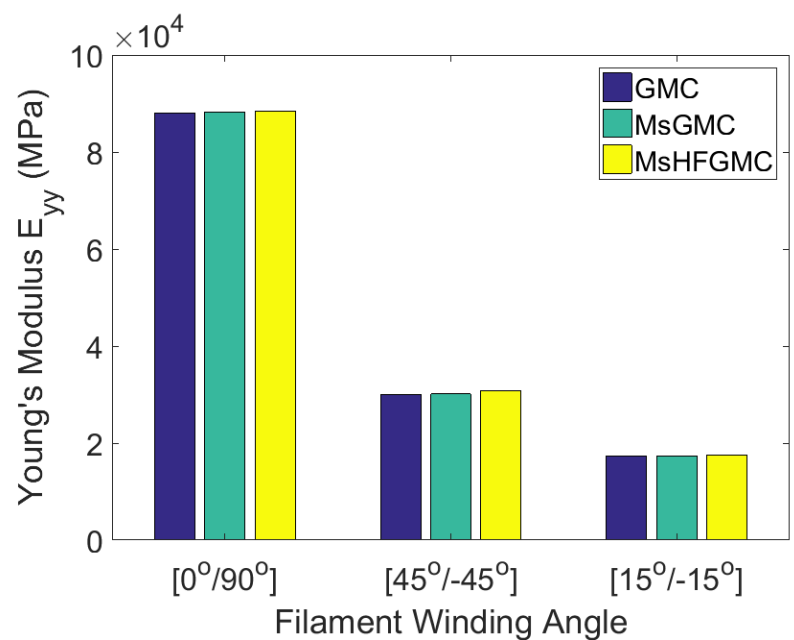

(b) $E_{y y}$.

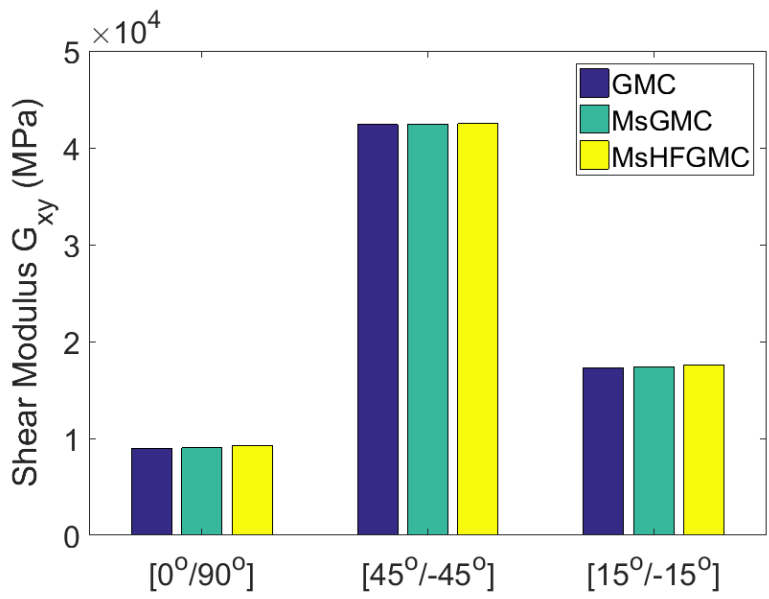

Filament Winding Angle

(c) $G_{x y}$.

Figure 8. Prediction of elastic properties of filament wound $\mathrm{C} / \mathrm{C}-\mathrm{SiC}$ at various winding angles 


\section{CONCLUSIONS}

Three models were developed for predicting the elastic properties of $\mathrm{C} / \mathrm{C}-\mathrm{SiC}$ manufactured using wet filament winding and LSI. These models utilized the GMC and HFGMC micromechanics theories across multiple length scales. The first model consisted of a concurrent length scale representation of all three phases within the $\mathrm{C} / \mathrm{C}-\mathrm{SiC}$ composite RUC an utilized GMC for localization and homogenization. The second scale incorporated two separate length scales: a mesoscale containing the $\mathrm{SiC}$ matrix and homogenized $\mathrm{C} / \mathrm{C}$ bundle, and a microscale containing the $\mathrm{C}$ fiber and amorphous $\mathrm{C}$ matrix. Communication between the scales was accomplished in a two-way coupling, synergistic manner (i.e., concurrent in time, but hierarchical in space) through localization and homogenization with GMC. The final model utilized HFGMC to calculated the homogenized properties of the microscale RUC for use in the constitutive relationship of the $\mathrm{C} / \mathrm{C}$ bundle at the mesoscale via a bottom-up, one way coupling, hierarchical multiscale framework.

All three multiscale models were used to predict the effective properties of an RUC within a woven $\mathrm{C} / \mathrm{C}$-SiC composite, and showed excellent agreement with analogous FEM predictions available in the literature. Furthermore, the run time for all simulations was less than 10 seconds using a single CPU. The same material properties and geometric parameters for the RUCs were used to predict the elastic properties of filament wound $\mathrm{C} / \mathrm{C}-\mathrm{SiC}$ with three different winding angles. Predictions, by all three models, for two out of the three configurations exhibited excellent agreement with experimental data. The error exhibited in the predictions for the third configuration $\left[15^{\circ} / 15^{\circ}\right]$ support the micro-graphical evidence that the $\mathrm{C} / \mathrm{C}$-SiC microstructure changes depending on the filament winding angle. To capture this the RUCs used for

this particular angle would need to be altered based upon measurements taken at the microscale.

The effect of both free $\mathrm{Si}$ and $\mathrm{C}$ matrix porosity on the elastic properties of a $\mathrm{C} / \mathrm{C}-\mathrm{SiC}$ composite can be resolved by adjusting the effective properties of the homogenized representation of $\mathrm{SiC}$ and amorphous $\mathrm{C}$. However, this will not produce the same effect on the local fields within the composite. The local fields are the main driving force for any damage initiation or inelasticity that may arise and must be considered if accurate non-linear predictions are required. The multiscale analysis presented in this work shows that the addition of free $\mathrm{Si}$ or $\mathrm{C}$ matrix porosity tends to reduce the average effective stress within the $\mathrm{C}$ matrix phase of the composite. While the free $\mathrm{Si}$ also reduces the maximum effective stress, locally within the $\mathrm{C}$ matrix, the presence of pores increases this value and serves as a stress riser. Full-field micromechanics theories are necessary to capture such effects.

It has been demonstrated that, multiscale modeling frameworks utilizing GMC and HFGMC are in place for predicting the elastic properties of filament wound $\mathrm{C} / \mathrm{C}$ $\mathrm{SiC}$ at every length scale. Additional phases can be included in the models, and it is possible to represent the microstructure as a function of the filament winding angle (although this was not demonstrated here). With this framework in place, full-scale, filament wound $\mathrm{C} / \mathrm{C}-\mathrm{SiC}$ structures can be tailored and optimized to meet stiffness and strength requirements in a computationally efficient manner. In addition, other aspects of the filament wound $\mathrm{C} / \mathrm{C}$-SiC composite can be modeled including thermal 
properties and effects, processing, and damage evolution.

\section{ACKNOWLEDGEMENTS}

Dr. Pineda would like to acknowledge, the Theodore von Kármaán Fellowship (GS069), and the Alexander von Humboldt Fellowship for partial funding this work. Also, Dr. Simon is thankful for the support of the Ministry of Innovation, Science, and Research of the state of North Rhine-Westphalia. The authors would also like to thank Fabian Breede from DLR for his input regarding this work.

Financial support has been provided by the German Research Foundation (DFG) in the framework of CRC/Translogic 40 Fundamental Technologies for the Development of Future Space-Transport-System Components under High Thermal and Mechanical Loads (TPD3)

\section{REFERENCES}

1. Schulte-Fischedick, J., A. Zern, J. Mayer, M. Rühle, M. Frieß, et al. 2002, "The morphology of silicon carbide in C/C-SiC composites," Mater. Sci. Eng. A, 332:146-152.

2. Krenkel, W. 2005, "Carbon fibre reinforced silicon carbide composites (C/SiC, C/C$\mathrm{SiC})$," in Handbook of Ceramic Composites, N. Bansal, ed., Springer, pp. 117-148.

3. Krenkel, W. 2004, "Carbon fiber reinforced CMC for high-performance structures," Int. J. Appl. Ceram. Technol., 1(2):188-200.

4. Krenkel, W. 2008, "Microstructure tailoring of C/C-SiC composites," in 27th Annual Cocoa Beach Conference on Advanced Ceramics and Composites -B: Ceramic Engineering and Science Proceedings, W. M. Kriven, ed., John Wiley \& Sons, pp. 471-476.

5. Sheikh, M. A., D. R. Hayhurst, S. C. Taylor, and R. Taylor. 2009, "Experimental investigation of the effect of mechanical loading on thermal transport of ceramic composites," J. Multiscale Model., 1(3-4):403-431.

6. Hofmann, S., B. Öztuürk, D. Koch, and H. Voggenreiter. 2012, "Experimental and numerical evaluation of bending and tensile behavior of carbon-fiber reinforced SiC," Compos. Part A, 43:1877-1885.

7. Breede, F., M. Frieß, R. Jemmali, D. Koch, and H. Voggenreiter. 2013, "Mechanical and microstructural characterization of $\mathrm{C} / \mathrm{C}-\mathrm{SiC}$ manufactured via triaxial and biaxial preforms," in Mechanical Properties of Performance of Engineering Ceramics and Composites VII, D. Singh and J. Salem, eds., The American Ceramic Society, pp. 183-194.

8. Rak, Z. S. 2002, "C $\mathrm{C}_{f} / \mathrm{SiC}$ composites by a low-cost manufacturing process," Key Eng. Mater., 206-213:1049-1052.

9. Schmidt, S., S. Beyer, H. Knabe, H. Immich, R. Meistring, et al. 2004, "Advanced ceramic matrix composite materials for current and future propulsion technology applications," Acta Astronaut., 55:409-420. 
10. Blacklock, M. and D. R. Hayhurst. 2012, "Initial elastic properties of unidrectional ceramic matrix composite fiber tows," J. Appl. Mech., 79:1-11.

11. Weigel, N., B. Kröplin, and D. Dinker. 1999, "Micromechanical modeling of damage and failure mechanisms in C/C-SiC," Comput. Mater. Sci., 16:120-132.

12. Weigel, N., D. Dinkler, and B. H. Kröplin. 2001, "Micromechanically based continuum damage mechanics material laws for fiber-reinforced ceramics," Comput. Struct., 79:2277-2286.

13. Baste, S. 2001, "Inelastic behavior of ceramic-matrix composites," Compos. Sci. Technol., 61:2285-2297.

14. Tang, C., M. Blacklock, and D. R. Hayhurst. 2009, "Uni-axial stress-strain response of thermal conductivity degradation of ceramic matrix composite fibre tows," Proc. R. Soc. A, 465:2849-2876.

15. Tang, C., M. Blacklock, and D. R. Hayhurst. 2010, "Stress-strain response and thermal conductivity degradation of ceramic matrix composite fiber tows in $0-90^{\circ}$ uni-directional and woven composites," J. Compos. Mater., 45(14):1461-1482.

16. Blacklock, M. and D. R. Hayhurst. 2011, "Multi-axial failure of ceramic matrix composite fiber tows," J. Appl. Mech., 78(3):1-10.

17. Shi, Y., S. Hofmann, R. Jemmali, S. Hackemann, and D. Koch. 2014, "Determination of elastic properties for a wound oxide ceramic composite," J. Ceram. Sci. Technol., 5(1):31-38.

18. Breede, F., S. Hofmann, E. Klatt, and S. Denis. 2011, "Influence of fiber orientation on the mechanical properties and microstructure of $\mathrm{C} / \mathrm{C}-\mathrm{SiC}$ composite plates produced by wet filament winding technique," in Processing and Properties of Advanced Ceramics and Composites III, N. P. Bansal, J. P. Singh, and J. Lamon, eds., The American Ceramic Society, pp. 3-10.

19. Breede, F., R. Jemmali, H. Voggenreiter, and D. Koch. 2014, "Design and testing of a $\mathrm{C} / \mathrm{C}-\mathrm{SiC}$ nozzle extension manufactured via filament winding technique and liquid silicon infiltration," in Design, Development, and Application of Structural Ceramics, Composites, and Nanomaterials, Volume 244, D. Singh, D. Zhu, W. M. Kriven, and S. Mathur, eds., The American Ceramic Society and John Wiley \& Sons, pp. 3-14.

20. Aboudi, J., S. M. Arnold, and B. A. Bednarcyk. 2013, Micromechanics of Composite Materials: A Generalized Multiscale Analysis Approach, Elsevier, Inc.

21. Liu, K. C., A. Chattopadhyay, B. A. Bednarcyk, and S. M. Arnold. 2011, "Efficient multiscale modeling framework for triaxially braided composites using the generalized method of cells," J. Aerospace Eng., 24:162-169.

22. Breede, F., D. Koch, and M. Frieß. 2012, "Development and characterisation of novel $\mathrm{C} / \mathrm{C}$-SiC material using LSI method and effective fiber preform techniques for rocket nozzle extensions," SFB/TRR 40 - Annual Report 2012.

23. Paley, M. and J. Aboudi. 1992, "Micromechanical Analysis of Composites by the Generalized Cells Model," Mechanics of Materials, 14(2):127-139. 
24. Arnold, S. M., M. J. Pindera, and T. E. Wilt. 1996, "Influence of fiber architecture on the inelastic response of metal matrix composites," Int. J. Plasticity, 12(4):507-545.

25. Jones, R. M. 1999, Mechanics of Composite Materials, 2nd Ed., Taylor and Francis, Inc., Philadelphia. 